\title{
Changing the Wound: Covalent Immobilization of Epidermal Growth Factor
}

VijayKrishna Raghunathan ${ }^{1,9,10 \dagger}$, Shin Ae Park ${ }^{1,11 \dagger,}$, Nihar M. Shah ${ }^{1,12, \dagger}$, Christopher M. Reilly ${ }^{2}$, Leandro Teixeira ${ }^{3}$, Richard Dubielzig ${ }^{3}$, Yow-Ren Chang ${ }^{1}$, Monica Motta ${ }^{1}$, Michael J. Schurr ${ }^{4}$, Jonathan F. McAnulty ${ }^{5}$, R. Rivkah Isseroff ${ }^{6}$, Nicholas L. Abbott ${ }^{7^{*}}$, Christopher J. Murphy ${ }^{1,8^{*}}$

\section{Affiliations:}

${ }^{1}$ Department of Surgical and Radiological Sciences, School of Veterinary Medicine, University of California Davis, One Shields Avenue, Davis, CA, 95616, USA

${ }^{2}$ Department of Pathology, Microbiology \& Immunology, School of Veterinary Medicine, University of California Davis, One Shields Avenue, Davis, CA, 95616, USA

${ }^{3}$ Dept. of Pathobiological Sciences, School of Veterinary Medicine, University of WisconsinMadison, Madison, WI, 53706, USA

${ }^{4}$ Divison of General Surgery, Mountain Area Health Education Center, 509 Biltmore Avenue, Asheville, NC, 28803, USA

${ }^{5}$ Department of Surgical Sciences, School of Veterinary Medicine, University of WisconsinMadison, 2015 Linden Dr, Madison, WI, 53706, USA

${ }^{6}$ Department of Dermatology, UC Davis School of Medicine, University of California Davis, Sacramento, CA, 95817, USA

${ }^{7}$ Smith School of Chemical and Biomolecular Engineering, Cornell University, 1 Hoy Plaza, Ithaca, NY, 14853 USA

${ }^{8}$ Department of Ophthalmology \& Vision Science, School of Medicine, University of California Davis, One Shields Avenue, Davis, CA, 95817, USA

${ }^{9}$ Department of Basic Sciences, College of Optometry, 4901 Calhoun Rd, University of Houston, Houston, TX, 77204, USA

${ }^{10}$ Biomedical Engineering, Cullen College of Engineering, University of Houston, Houston, TX, 77204, USA

${ }^{11}$ Department of Veterinary Clinical Sciences, College of Veterinary Medicine, Purdue University, 625 Harrison Street, West Lafayette, IN, 47907, USA

${ }^{12}$ Medtronic Diabetes, 18000 Devonshire St, Northridge, CA, USA 91325-1219

${ }^{\dagger}$ Authors contributed equally to this work.

“Corresponding authors: nla34@cornell.edu, cjmurphy@ucdavis.edu

Supplementary materials: Supplementary figures: 6 
A
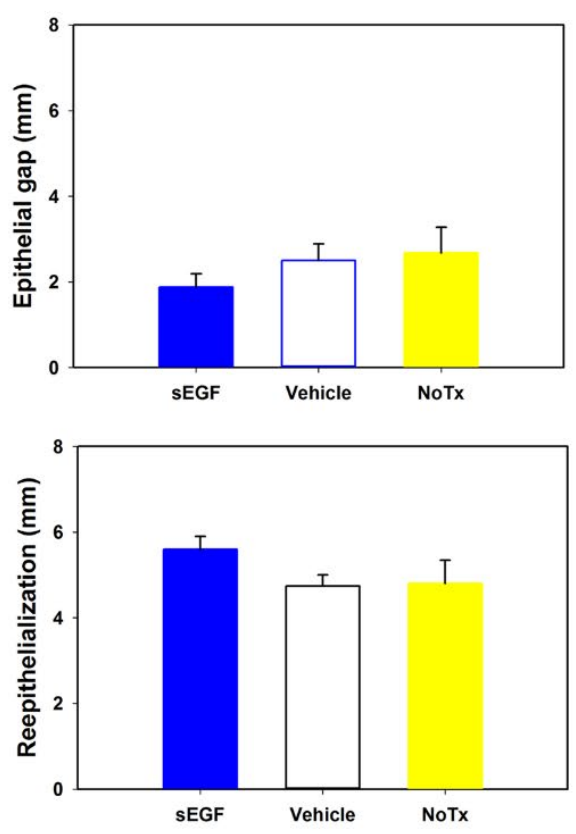

B

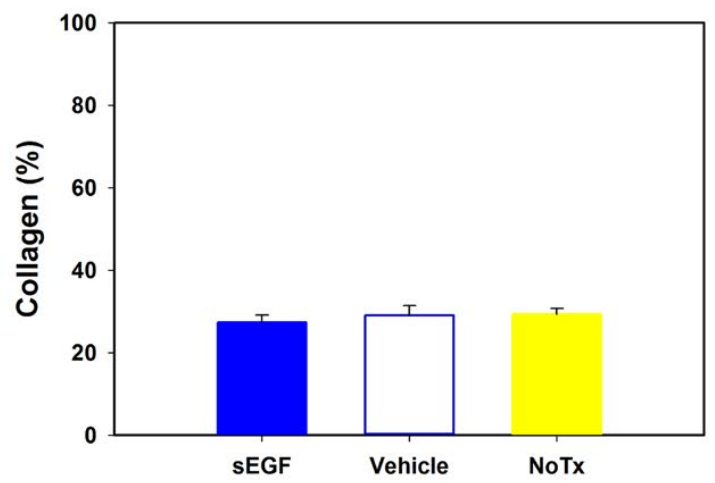

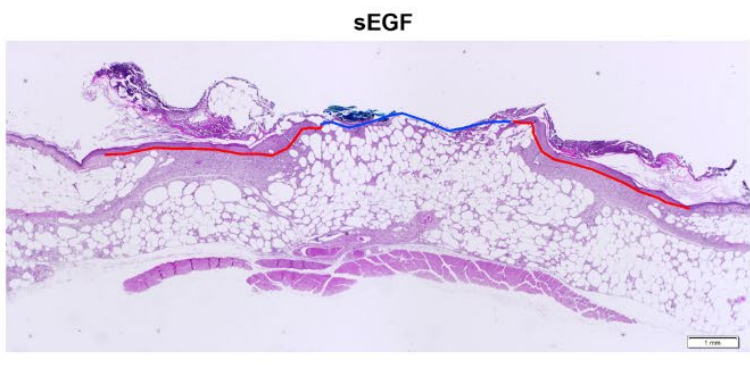

Vehicle

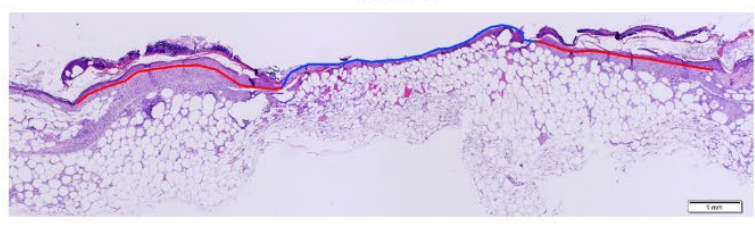

NoTx

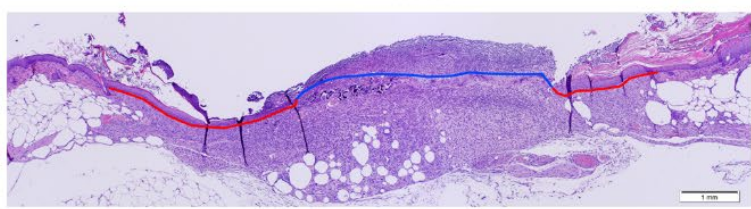

sEGF

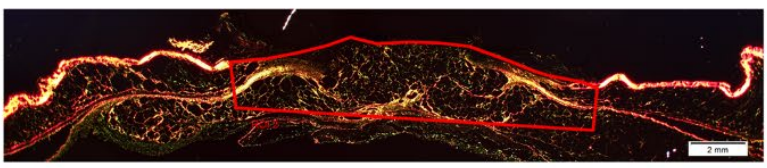

Vehicle

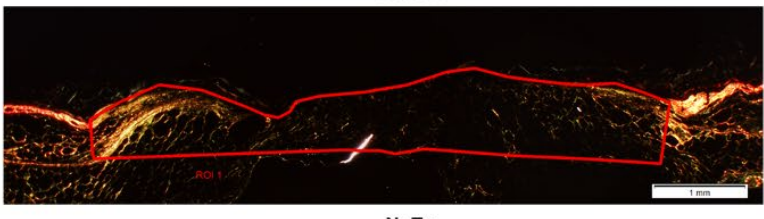

NoTx

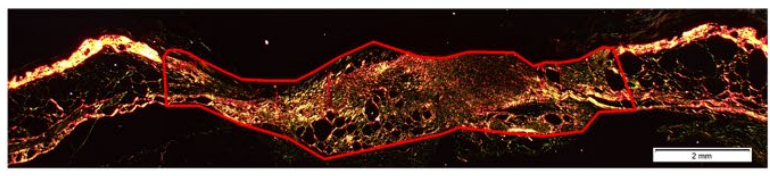

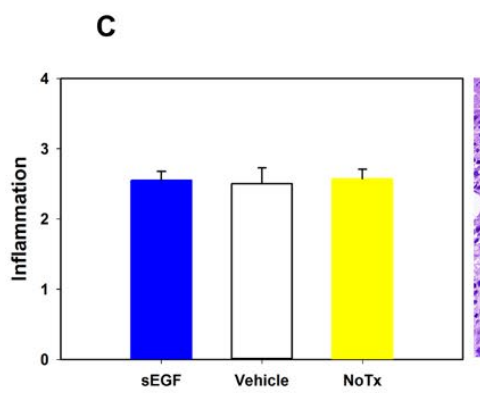
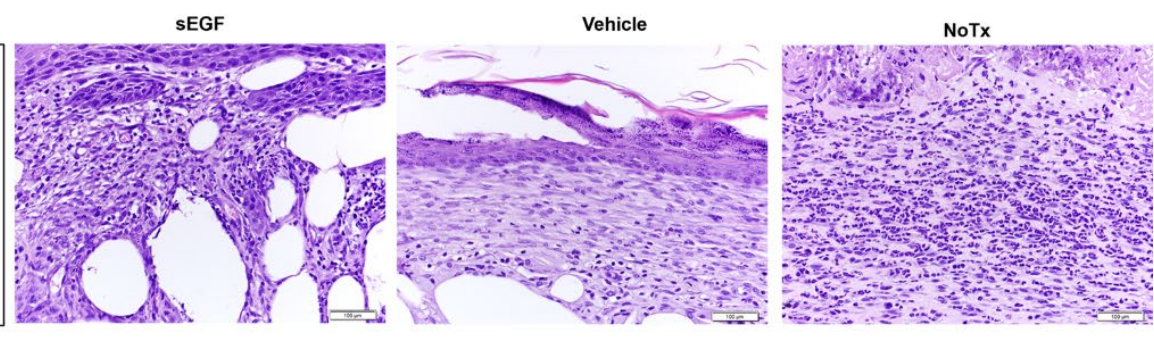
Figure S1: Histopathological evaluations of a wound bed from a $\mathrm{db} / \mathrm{db}$ mouse treated with/without topically applied EGF (twice daily) over 11 days upon wounding. Scale Bars $=500$ $\mu \mathrm{m}$. (A) Measurement of epithelial gap (blue line) and reepithelialization (red line). The epithelial gap was defined as the distance between the advancing edges of keratinocyte migration measured in millimeters. Length of reepithelialization was defined as the length of the layer of proliferating keratinocytes covering the wound area. This value was obtained by measuring $s$ the distance between the free edge of the keratinocyte layer and the base where the cells were still associated with native, non-affected dermal tissue The final value was the sum of distance in millimeters of both sides. H\&E staining. Graphs indicate the mean \pm standard deviation of epithelial gap / re-epithelialized measure on surface wounds after 11 days with or without treatments. (B) Measurement of the fibrovascular dermal proliferation in the wound bed. Using picrosirius red stain under polarized light the bright collagen fibers of the wound bed are highlighted and automatically measured by the software. The final data is expressed as a percentage of outlined wound area comprised of collagen. Graphs indicate the mean \pm standard deviation of \% collagen measured in surface wounds after 11 days with or without treatments. (C) Cross linked EGF elicited less inflammation. The inflammatory response was assessed using a semi-quantitative scoring system ranging from 0 to 4 where 0 indicates no inflammation, 1 indicates $0-25 \%$ of the wound area affected, 2 indicates $25-50 \%$ of the wound area affected, 3 indicates $50-75 \%$ of the wound area affected, and 4 indicates $75 \%$ of the wound area affected.. H\&E staining. Graphs indicate the mean \pm standard deviation of inflammation score. 


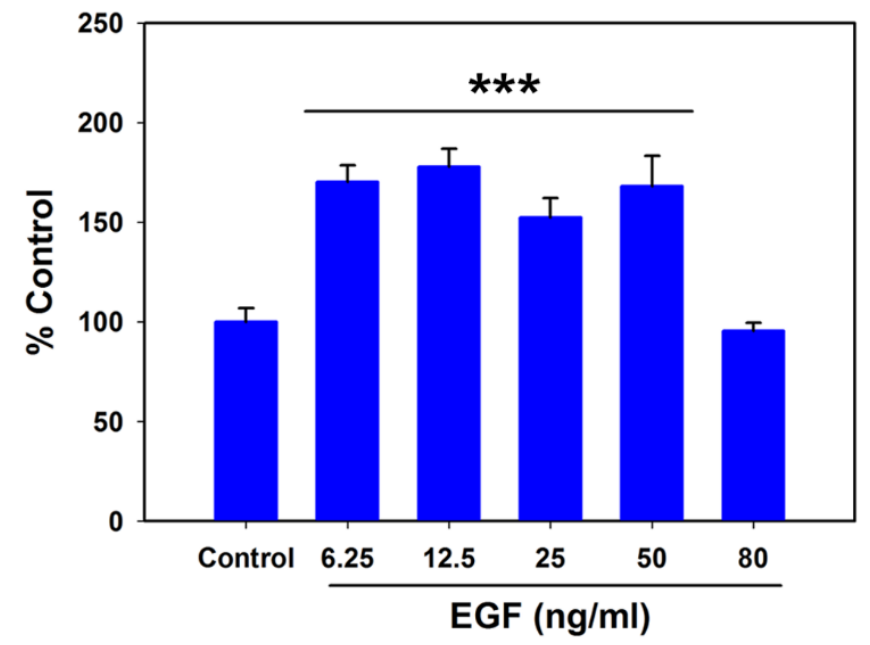

Figure S2: In vitro proliferation rates of spontaneously immortalized human keratinocytes (HaCaT cells), cultured in growth medium containing $1 \%(\mathrm{v} / \mathrm{v})$ serum (FBS), treated with $0-80$ $\mathrm{ng} / \mathrm{ml}$ soluble EGF. Results are mean \pm standard deviation, ${ }^{* * *} \mathrm{p}<0.001$ (ANOVA followed by Dunnett's multiple comparison test) compared with control cultures. Soluble EGF increased cell proliferation. 


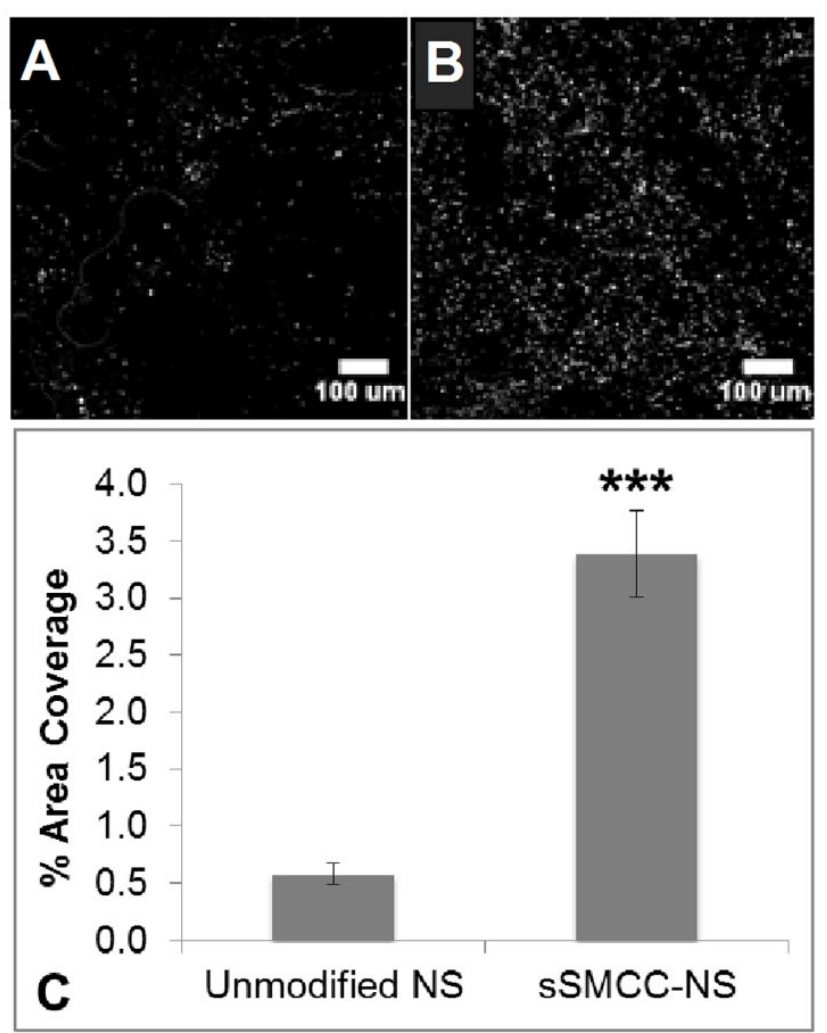

Figure S3: Heterobifunctional crosslinker sulfo-SMCC significantly improves attachment of PLGA nanospheres (NS) on Porcine dermis tissue. Fluorescent micrographs of Porcine dermis tissue samples treated with (A) unmodified NS, and (B) sulfo-SMCC activated NS. (C) Preactivation of the NS with sulfo-SMCC improved their attachment to Porcine dermis tissue by $83 \% . n=6$ samples per treatment group, 4 images per sample; ${ }^{* * *} p<0.001$. 


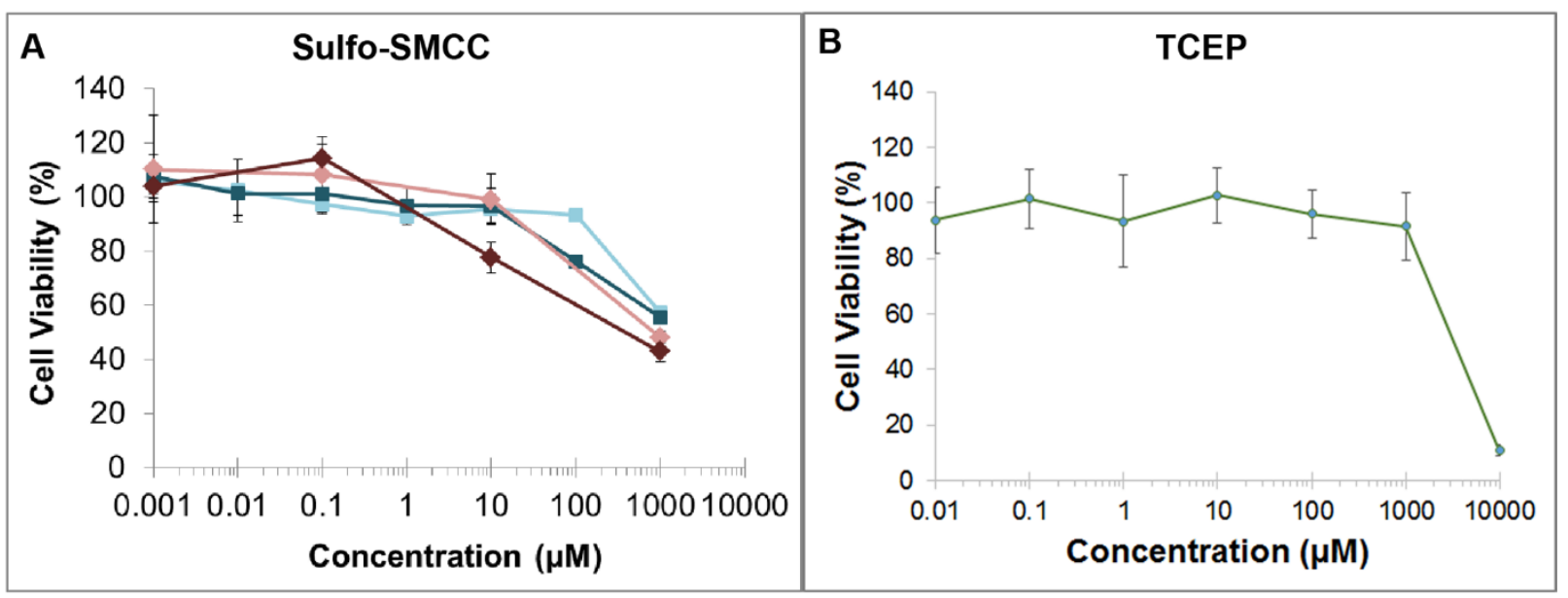

Figure S4: HaCaT and HMVEC viability is differentially affected by the hydrolyzed state and concentration of (A) sulfo-SMCC or (B) TCEP. In vitro screening of HaCaT $(\bullet)$ and HMVEC (ם) viability by calcein-AM assay following $1 \mathrm{~h}$ exposure to fresh (light pink $\diamond$ and light blue $\mathbf{\square}$ ), and hydrolyzed (dark pink $\diamond$ and dark blue $\mathbf{0}$ ), solutions of in DPBS, at a concentration range of $0.001 \mu \mathrm{M}$ to $10000 \mu \mathrm{M}(1 \mathrm{nM}$ to $10 \mathrm{mM})$. Maximum concentration of sulfo-SMCC was $1 \mathrm{mM}$ due to lower solubility in DPBS. For HaCaTs, $\mathrm{n}=10$ for each state and concentration. For HMVECs, $n=5$ for each state and concentration. Error bars are \pm SEM. 
A

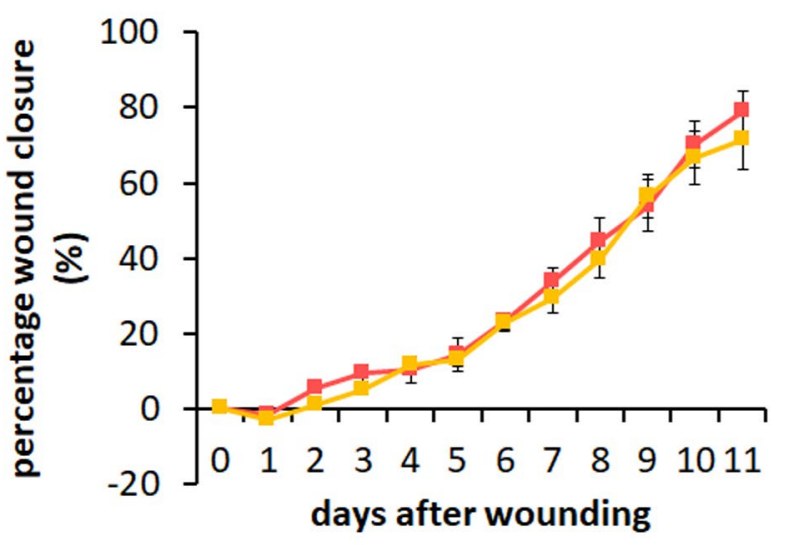

$=-$ CXEGF-1x $\rightarrow-$ NoTx
B

wound healing rate

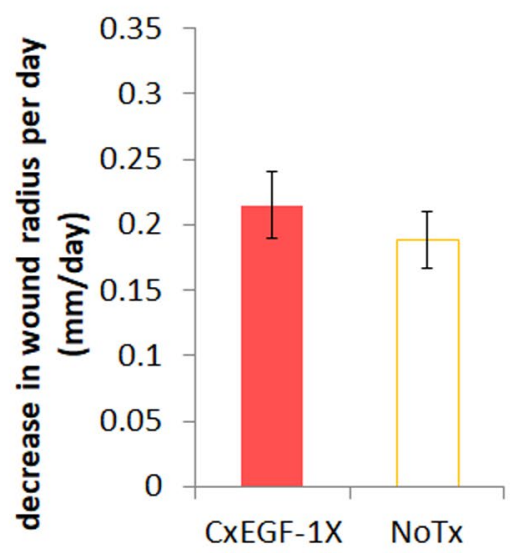

C

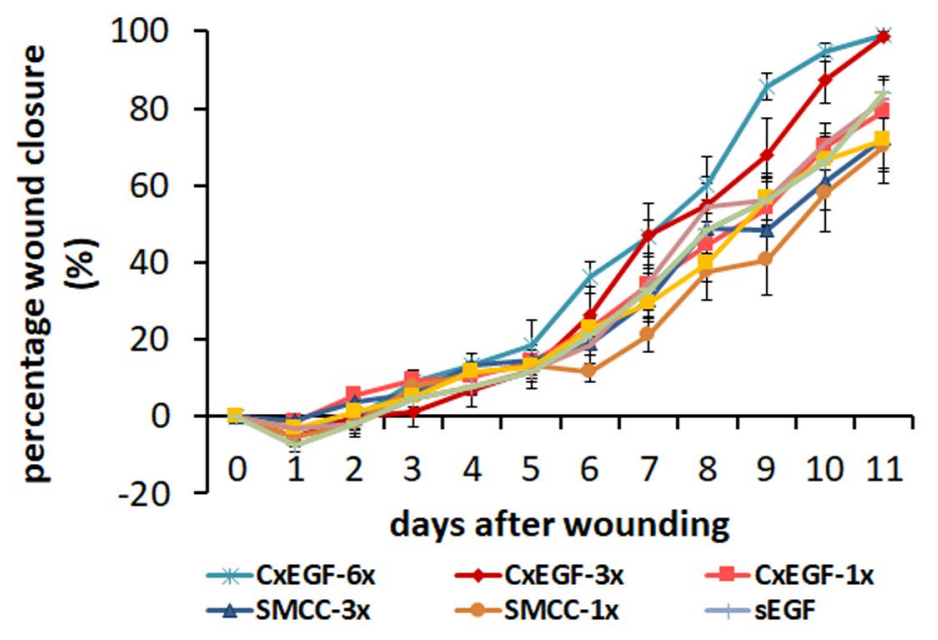

Figure S5: (A) Percentage wound closure and (B) wound healing rate were not significantly different between no treatment groups and once only crosslinked EGF (CxEGF-1x) group suggesting repeated treatments may be required. (C) Graph demonstrates percentage wound closure of all experimental groups conducted in this study. 

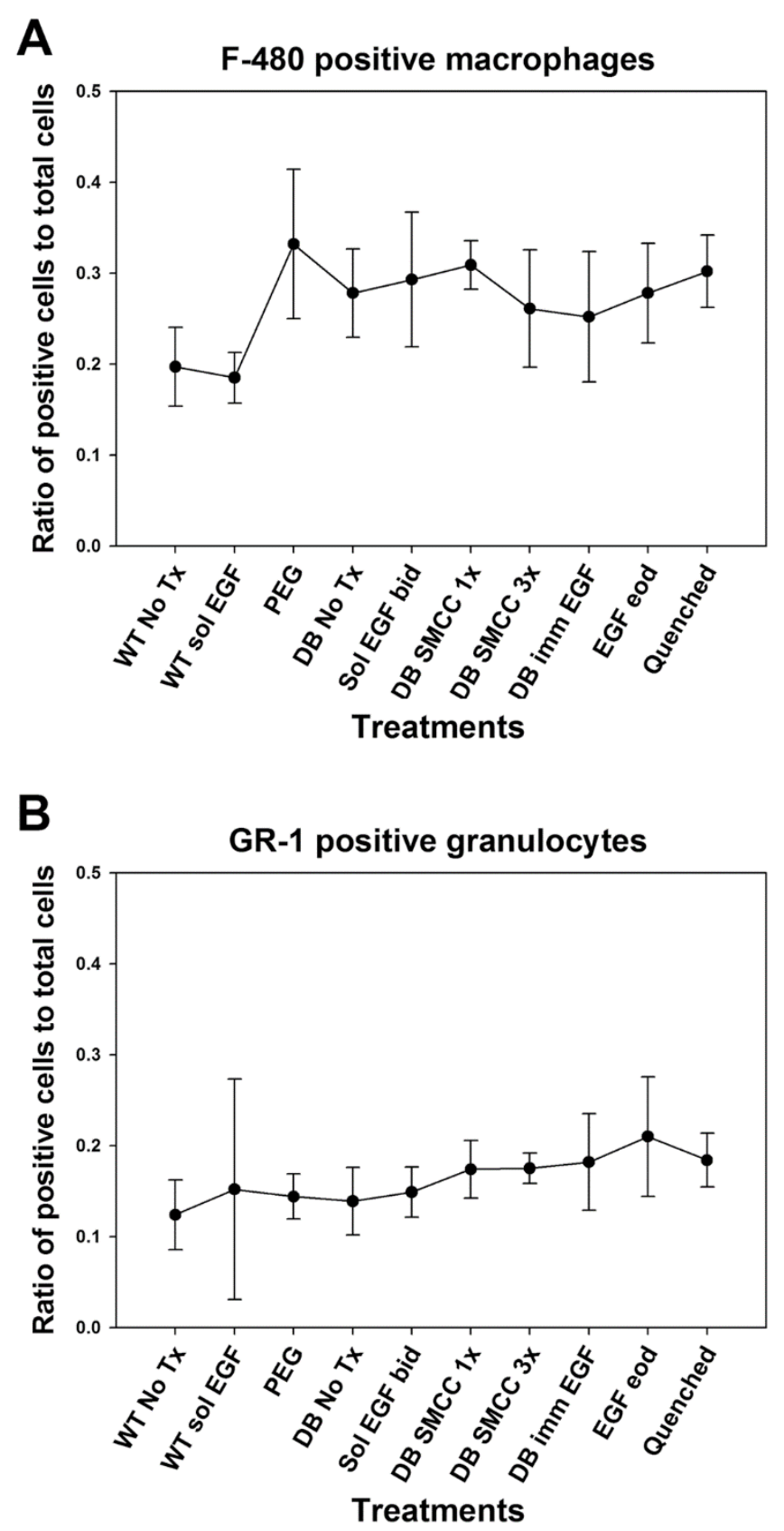

Figure S6: Percentage distribution of inflammatory cells in wound bed of diabetic mice at the end of 11 days. No statistically significant differences in (A) F-480 positive macrophages or (B) GR1 positive granulocytes were detected in wound bed from a db/db mouse treated with/without crosslinked EGF after 11 days upon wounding. Results are mean \pm standard deviation of the ratio of positive cells to total number of cells in he wound bed. 